\title{
Exact Solutions for a Class of Variable Coefficients \\ Nonlinear Evolution Equations
}

\author{
Haiming $\mathrm{Fu}$ \\ Mathematics Department \\ Yunnan University \\ No. 2 Cuihu Road, Kunming 650091, China \\ E-mail:haimingfu@163.com
}

\begin{abstract}
A class of Variable Coefficients nonlinear evolution equations $u_{t}+\left[\alpha(t)+\beta(t) u^{2}\right] u^{2} u_{x}+\gamma(t) u_{x x x}=0$ With $\alpha(t)>0, \beta(t)<0$. By using the general process of working out the exact solution of the nonlinear evolution equations in the homogeneous balance method, and the exact solutions of the equations are obtained.
\end{abstract}

Keywords: Nonlinear equation, Homogeneous balance method, Solitary wave solution

\section{Introduction}

Nonlinear science is the nucleus of modern science, in natural science, some descriptions concern soliton equation, at soliton theory, the solve method of soliton equation is the importance of question research. Because of new methods' appearing, not only the equations hard to solve before have been solved, but also discover the soliton equation have important physics significance. Recently, homogeneous balance method successful solves some of soliton equations.

\section{Exact Solutions of Solitary Wave Solution}

We consider a class of Variable Coefficients nonlinear evolution equations as following:

$$
u_{t}+\left[\alpha(t)+\beta(t) u^{2}\right] u^{2} u_{x}+\gamma(t) u_{x x x}=0
$$

With $\alpha(t)>0, \beta(t)<0$. Suppose (1) has the solution

$$
u(x, t)=f^{\prime} \varphi_{x}^{m} \varphi_{t}^{n}
$$

with $f=f(\varphi)$ and $\varphi=\varphi(x, t)$. Then

$$
\begin{aligned}
& u^{2} u^{2} u_{x}=f^{, 2} \varphi_{x}^{2 m} \varphi_{t}^{2 n} f^{, 2} \varphi_{x}^{2 m} \varphi_{t}^{2 n}\left(f^{\prime \prime} \varphi_{x}^{m+1} \varphi_{t}^{n}+m f^{\prime} \varphi_{x}^{m-1} \varphi_{t}^{n} \varphi_{x x}+n f^{\prime} \varphi_{x}^{m} \varphi_{t}^{n-1} \varphi_{t x}\right) \\
& u_{x x x}=f^{(4)} \varphi_{x}^{m+3} \varphi_{t}^{n}
\end{aligned}
$$

Using homogeneous balance method, we get

$$
\left\{\begin{array} { c } 
{ m + 3 = 2 m + 2 m + m + 1 } \\
{ 2 n + 2 n + n = n }
\end{array} , \text { further } \left\{\begin{array}{c}
m=\frac{1}{2} \\
n=0
\end{array}\right.\right.
$$

Let

$$
u(x, t)=a(t) \varphi_{x}^{\frac{1}{2}} \varphi^{-\frac{1}{2}} \quad(a(t) \neq 0)
$$

Thus

$$
\begin{aligned}
& u_{t}=a^{\prime}(t) \varphi_{x}^{\frac{1}{2}} \varphi^{-\frac{1}{2}}+\frac{1}{2} a(t) \varphi_{x}^{-\frac{1}{2}} \varphi_{x t} \varphi^{-\frac{1}{2}}-\frac{1}{2} a(t) \varphi_{x}^{\frac{1}{2}} \varphi^{-\frac{3}{2}} \varphi_{t} \\
& u^{2}=a^{2}(t) \varphi_{x} \varphi^{-1} \\
& u_{x}=\frac{1}{2} a(t) \varphi_{x}^{-\frac{1}{2}} \varphi_{x x} \varphi^{-\frac{1}{2}}-\frac{1}{2} a(t) \varphi_{x}^{\frac{1}{2}} \varphi^{-\frac{3}{2}} \varphi_{x}
\end{aligned}
$$




$$
\begin{gathered}
u_{x x}=\frac{3}{4} a(t) \varphi_{x}^{\frac{5}{2}} \varphi^{-\frac{5}{2}}-\frac{3}{4} a(t) \varphi_{x}^{\frac{1}{2}} \varphi^{-\frac{3}{2}} \varphi_{x x}-\frac{1}{4} a(t) \varphi_{x}^{\frac{1}{2}} \varphi_{x}^{-\frac{3}{2}} \varphi_{x x}+\frac{1}{2} a(t) \varphi_{x}^{-\frac{1}{2}} \varphi^{-\frac{1}{2}} \varphi_{x x x}-\frac{1}{4} a(t) \varphi_{x}^{-\frac{3}{2}} \varphi^{-\frac{1}{2}} \varphi_{x x}^{2} \\
=\frac{3}{4} a(t) \varphi_{x}^{\frac{5}{2}} \varphi^{-\frac{1}{2}}-a(t) \varphi_{x}^{\frac{1}{2}} \varphi^{-\frac{3}{2}} \varphi_{x x}+\frac{1}{2} a(t) \varphi_{x}^{-\frac{1}{2}} \varphi^{-\frac{1}{2}} \varphi_{x x x}-\frac{1}{4} a(t) \varphi_{x}^{-\frac{3}{2}} \varphi^{-\frac{1}{2}} \varphi_{x x}^{2}
\end{gathered}
$$

$u_{x x x}=\frac{15}{8} a(t) \varphi_{x}^{\frac{3}{2}} \varphi_{x x} \varphi^{-\frac{5}{2}}-\frac{15}{8} a(t) \varphi_{x}^{\frac{7}{2}} \varphi^{-\frac{7}{2}}-\frac{3}{8} a(t) \varphi_{x}^{-\frac{1}{2}} \varphi_{x x}^{2} \varphi^{-\frac{3}{2}}-\frac{3}{4} a(t) \varphi_{x}^{\frac{1}{2}} \varphi_{x x x} \varphi^{-\frac{3}{2}}+$

$\frac{9}{8} a(t) \varphi_{x}^{\frac{3}{2}} \varphi_{x x} \varphi^{-\frac{5}{2}}-\frac{1}{8} a(t) \varphi_{x}^{-\frac{1}{2}} \varphi_{x x}^{2} \varphi^{-\frac{3}{2}}-\frac{1}{4} a(t) \varphi_{x}^{\frac{1}{2}} \varphi_{x x x} \varphi^{-\frac{3}{2}}-\frac{3}{8} a(t) \varphi_{x}^{\frac{3}{2}} \varphi_{x x} \varphi^{-\frac{5}{2}}-$

$\frac{1}{4} a(t) \varphi_{x}^{-\frac{3}{2}} \varphi_{x x} \varphi_{x x x} \varphi^{-\frac{1}{2}}+\frac{1}{2} a(t) \varphi_{x}^{-\frac{1}{2}} \varphi_{x x x x} \varphi^{-\frac{1}{2}}-\frac{1}{4} a(t) \varphi_{x}^{\frac{1}{2}} \varphi_{x x x} \varphi^{-\frac{3}{2}}+\frac{3}{8} a(t) \varphi_{x}^{-\frac{5}{2}} \varphi_{x x}^{3} \varphi^{-\frac{1}{2}}-$

$\frac{1}{2} a(t) \varphi_{x}^{\frac{3}{2}} \varphi_{x x x} \varphi^{-\frac{1}{2}} \varphi_{x x}+\frac{1}{8} a(t) \varphi_{x}^{-\frac{1}{2}} \varphi_{x}^{2} \varphi^{-\frac{3}{2}}$

$=\frac{21}{8} a(t) \varphi_{x}^{\frac{3}{2}} \varphi_{x x} \varphi^{-\frac{5}{2}}-\frac{15}{8} a(t) \varphi_{x}^{\frac{7}{2}} \varphi^{-\frac{7}{2}}-\frac{3}{8} a(t) \varphi_{x}^{-\frac{1}{2}} \varphi_{x x}^{2} \varphi^{-\frac{3}{2}}-\frac{5}{4} a(t) \varphi_{x}^{\frac{1}{2}} \varphi_{x x x} \varphi^{-\frac{3}{2}}-$

$$
\frac{3}{4} a(t) \varphi_{x}^{-\frac{3}{2}} \varphi_{x x} \varphi_{x x x} \varphi^{-\frac{1}{2}}+\frac{1}{2} a(t) \varphi_{x}^{-\frac{1}{2}} \varphi_{x x x x} \varphi^{-\frac{1}{2}}+\frac{3}{8} a(t) \varphi_{x}^{-\frac{5}{2}} \varphi_{x x}^{3} \varphi^{-\frac{1}{2}}
$$

From (6),(7),(8),(10) and (1),we have

$\frac{1}{2} a(t) \varphi_{x}^{-\frac{1}{2}} \varphi_{x t} \varphi^{-\frac{1}{2}}-\frac{1}{2} a(t) \varphi_{x}^{\frac{1}{2}} \varphi^{-\frac{3}{2}} \varphi_{t}+a^{\prime}(t) \varphi_{x}^{\frac{1}{2}} \varphi^{-\frac{1}{2}}+\frac{1}{2} a^{3}(t) \alpha(t) \varphi_{x}^{\frac{1}{2}} \varphi_{x x} \varphi^{-\frac{3}{2}}+\frac{1}{2} a^{3}(t) \alpha(t) \varphi_{x}^{\frac{5}{2}} \varphi^{-\frac{5}{2}}+$ $\frac{1}{2} a^{5}(t) \beta(t) \varphi_{x}^{\frac{3}{2}} \varphi_{x x} \varphi^{-\frac{5}{2}}-\frac{1}{2} a^{5}(t) \beta(t) \varphi_{x}^{\frac{7}{2}} \varphi^{-\frac{7}{2}}+\frac{15}{8} a(t) \gamma(t) \varphi_{x}^{\frac{3}{2}} \varphi_{x x} \varphi^{-\frac{5}{2}}-\frac{15}{8} a(t) \gamma(t) \varphi_{x}^{\frac{7}{2}} \varphi^{-\frac{7}{2}}-$ $\frac{3}{8} a(t) \gamma(t) \varphi_{x}^{-\frac{1}{2}} \varphi_{x x}^{2} \varphi^{-\frac{3}{2}}-\frac{3}{4} a(t) \gamma(t) \varphi_{x}^{\frac{1}{2}} \varphi_{x x x} \varphi^{-\frac{3}{2}}+\frac{9}{8} a(t) \gamma(t) \varphi_{x}^{\frac{3}{2}} \varphi_{x x} \varphi^{-\frac{5}{2}}-\frac{1}{8} a(t) \gamma(t) \varphi_{x}^{-\frac{1}{2}} \varphi_{x x}^{2} \varphi^{-\frac{3}{2}}-$ $\frac{1}{4} a(t) \gamma(t) \varphi_{x}^{\frac{1}{2}} \varphi_{x x x} \varphi^{-\frac{3}{2}}-\frac{3}{8} a(t) \gamma(t) \varphi_{x}^{\frac{3}{2}} \varphi_{x x} \varphi^{-\frac{5}{2}}-\frac{1}{4} a(t) \gamma(t) \varphi_{x}^{-\frac{3}{2}} \varphi_{x x} \varphi_{x x x} \varphi^{-\frac{1}{2}}+\frac{1}{2} a(t) \gamma(t) \varphi_{x}^{-\frac{1}{2}} \varphi_{x x x x} \varphi^{-\frac{1}{2}}-$ $\frac{1}{4} a(t) \gamma(t) \varphi_{x}^{\frac{1}{2}} \varphi_{x x x} \varphi^{-\frac{3}{2}}+\frac{3}{8} a(t) \gamma(t) \varphi_{x}^{-\frac{5}{2}} \varphi_{x x}^{3} \varphi^{-\frac{1}{2}}-\frac{1}{2} a(t) \gamma(t) \varphi_{x}^{-\frac{3}{2}} \varphi_{x x x} \varphi^{-\frac{1}{2}} \varphi_{x x}+\frac{1}{8} a(t) \gamma(t) \varphi_{x}^{-\frac{1}{2}} \varphi_{x x}^{2} \varphi^{-\frac{3}{2}}=0$

Suppose (5) and (11) have the solutions as a type of follows

$$
\varphi(x, t)=1+e^{k(t) x+w(t)}
$$

Thus $\varphi_{x}=k(t) e^{k(t) x+w(t)}, \varphi_{x x}=k^{2}(t) e^{k(t) x+w(t)}, \varphi_{x x x}=k^{3}(t) e^{k(t) x+w(t)}, \varphi_{x x x x}=k^{4}(t) e^{k(t) x+w(t)}$,

$\varphi_{t}=\left[k^{\prime}(t) x+w^{\prime}(t)\right] e^{k(t) x+w(t)}, \varphi_{x t}=\left[k^{\prime}(t) x+k(t) k^{\prime}(t)+w^{\prime}(t) k(t)\right] e^{k(t) x+w(t)}$,

From (12) and (11), we have

$$
\begin{aligned}
& \left(\frac{k(t) e^{k(t) x+w(t)}}{1+e^{k(t) x+w(t)}}\right)^{\frac{7}{2}}\left[-\frac{1}{2} a^{5}(t) \beta(t)-\frac{15}{8} a(t) \gamma(t)\right]+ \\
& \left(\frac{k(t) e^{k(t) x+w(t)}}{1+e^{k(t) x+w(t)}}\right)^{\frac{5}{2}}\left[-\frac{1}{2} a^{3}(t) \alpha(t)+\frac{1}{2} a^{5}(t) \beta(t) k(t)+\frac{21}{8} a(t) \alpha(t) k(t)\right]+ \\
& \left(\frac{k(t) e^{k(t) x+w(t)}}{1+e^{k(t) x+w(t)}}\right)^{\frac{3}{2}}\left[-\frac{1}{2} a(t) \frac{k^{\prime}(t)+w^{\prime}(t)}{k(t)}+\frac{1}{2} a^{3}(t) k(t)-\frac{3}{8} a(t) k^{2}(t)-\frac{5}{4} a(t) \gamma(t) k^{2}(t)\right]+ \\
& \left(\frac{k(t) e^{k(t) x+w(t)}}{1+e^{k(t) x+w(t)}}\right)^{\frac{1}{2}}\left[\frac{1}{2} a(t)\left(k^{\prime}(t)+k(t) k^{\prime}(t)+k(t) w^{\prime}(t)\right)-\frac{3}{4} a(t) k^{3}(t) \gamma(t)+\right. \\
& \left.\frac{1}{2} a(t) \gamma(t) k^{2}(t)+a(t)+\frac{3}{8} a(t) \gamma(t) k^{3}(t)\right]=0
\end{aligned}
$$


Let the coefficient of $\left(\frac{k(t) e^{k(t) x+w(t)}}{1+e^{k(t) x+w(t)}}\right)^{\frac{7}{2}},\left(\frac{k(t) e^{k(t) x+w(t)}}{1+e^{k(t) x+w(t)}}\right)^{\frac{5}{2}},\left(\frac{k(t) e^{k(t) x+w(t)}}{1+e^{k(t) x+w(t)}}\right)^{\frac{3}{2}},\left(\frac{k(t) e^{k(t) x+w(t)}}{1+e^{k(t) x+w(t)}}\right)^{\frac{1}{2}}$ are zero in (13), we obtain:

$$
\begin{aligned}
& -\frac{1}{2} a^{5}(t) \beta(t)-\frac{15}{8} a(t) \gamma(t)=0 \\
& -\frac{1}{2} a^{3}(t) \alpha(t)+\frac{1}{2} a^{5}(t) k(t) \beta(t)+\frac{21}{8} a(t) k(t) \gamma(t)=0 \\
& -\frac{1}{2} a(t)\left[w^{\prime}(t)+k^{\prime}(t)\right]+\frac{1}{2} a^{3}(t) k^{2}(t) \alpha(t)-\frac{3}{8} a(t) k^{3}(t) \gamma(t)-\frac{5}{4} a(t) k^{3}(t) \gamma(t)=0
\end{aligned}
$$

$\frac{1}{2} a(t)\left[k^{\prime}(t)+k(t) k^{\prime}(t)+k(t) w^{\prime}(t)\right]+a^{\prime}(t)-\frac{3}{4} a(t) k^{3}(t) \gamma(t)+\frac{1}{2} a(t) k^{2}(t) \gamma(t)+$

$$
\frac{3}{8} a(t) k^{3}(t) \gamma(t)=0
$$

We get the solution of equations as following:

$$
\begin{aligned}
a(t) & = \pm \sqrt[4]{-\frac{15 \gamma(t)}{4 \beta(t)}} \\
k(t) & =-\frac{\alpha(t)}{3 \beta(t) \gamma(t)} \sqrt{-15 \beta(t) \gamma(t)} \\
w(t) & =\int\left[\frac{35}{36} \alpha^{3}(t) \gamma(t)-\frac{1}{3} \alpha^{\prime}(t) \beta(t) \gamma(t)-\frac{1}{6} \alpha(t) \beta^{\prime}(t) \gamma(t)-\frac{1}{6} \alpha(t) \beta(t) \gamma^{\prime}(t)\right] \frac{\sqrt{-15 \beta(t) \gamma(t)}}{\beta^{2}(t) \gamma^{2}(t)} d t
\end{aligned}
$$

From (19), (20) and (12),we have

$$
\begin{aligned}
\varphi(x, t)=1+\exp \left\{-\frac{\alpha(t) x}{3 \beta(t) \gamma(t)} \sqrt{-15 \beta(t) \gamma(t)}+\right. \\
\left.\qquad\left[\frac{35}{36} \alpha^{3}(t) \gamma(t)-\frac{1}{3} \alpha^{\prime}(t) \beta(t) \gamma(t)-\frac{1}{6} \alpha(t) \beta^{\prime}(t) \gamma(t)-\frac{1}{6} \alpha(t) \beta(t) \gamma^{\prime}(t)\right] \frac{\sqrt{-15 \beta(t) \gamma(t)}}{\beta^{2}(t) \gamma^{2}(t)} d t\right\}
\end{aligned}
$$

From (18),(21) and (5), we have the solutions of (1):

$$
\begin{aligned}
& u(x, t)= \pm \sqrt{-\frac{5 \alpha}{2 \beta}} \cdot \sqrt{\frac{\exp \left\{-\frac{\alpha \sqrt{-15 \beta \gamma}}{3 \beta \gamma} x+\int\left[\frac{35}{36} \alpha^{3} \gamma-\frac{1}{3} \alpha \beta \gamma-\frac{1}{6} \alpha\left(\beta^{\prime} \gamma+\beta \gamma^{\prime}\right)\right] \frac{\sqrt{-15 \beta \gamma}}{\beta^{2} \gamma^{2}} d t\right\}}{1+\exp \left\{-\frac{\alpha \sqrt{-15 \beta \gamma}}{3 \beta \gamma} x+\int\left[\frac{35}{36} \alpha^{3} \gamma-\frac{1}{3} \alpha \beta \gamma-\frac{1}{6} \alpha\left(\beta^{\prime} \gamma+\beta \gamma^{\prime}\right)\right] \frac{\sqrt{-15 \beta \gamma}}{\beta^{2} \gamma^{2}} d t\right\}}} \\
& \alpha=\alpha(t), \beta=\beta(t), \gamma=\gamma(t) .
\end{aligned}
$$

\section{References}

Bai Chenglin, Liu Xiqiang and Bai Chengjie. (1999). Multiple soliton solutions of the (2+1)-dimensional Broer-Kaup equations. Acta photonica sinica, 28, 1029-1030.

M. J. Ablowitz, P. A. Carkson, Solitons. (1991). Nonlinear Evolution and Inverse Scattering . Cambridge University Press, New York. pp.47-350.

M. R. Miura. (1978). Backlund Transformtion. Springer Verlag, Berlin. pp. 4-156.

Senthilvelan M. (2001). on the extended applications of homogeneous balance method. Applied Mathematics and Computation, 123, 381-388.

Wang M L, Zhou Y B. (1996). Application of a homogeneous balance method to exact solutions of nonlinear equations in mathematical physics. Phys Lett A, 216, 67-75. 